\title{
Non-consultant career grade doctors: the dependable backbone of genitourinary medicine?
}

G enitourinary medicine (GUM) is a specialty dependent on nonconsultant career grade (NCCG) doctors. The NCCG covers a range of grades, which include associate specialist, staff grade, hospital practitioner, and clinical assistant. Other non-standard grades can include trust grade, hospital assistant, and clinical medical officer, etc. (However, the Royal College of Physicians (RCP) only includes associate specialists and staff grades in their definition of NCCG.) These posts can range from a single session to full time. Clinical background varies widely, which can positively enhance the diversity of clinical skills within the GUM team.

It is recognised that NCCGs do a significant proportion of the clinical GUM work. Data from the Association of Genitourinary Medicine (AGUM) in 2000 suggested that 592 doctors undertook 1685 GUM clinical sessions each week in the United Kingdom. More recent unpublished data from the GUM NCCG Group have confirmed that at least 419 doctors are working in the specialty as NCCG doctors, of whom 34 are associate specialists, 55 are staff grades (that is, 86 who fit the RCP definition of NCCG), 280 are clinical assistants, and another 50 whose grades were unspecified. Of the 280, 134 are clinical assistants in GUM only without other posts. (By comparison there are currently 266 whole time equivalent GUM consultants in the United Kingdom 2003 Census of Consultant Physicians, November 2004.)

In the past, NCCGs have been described as "a reliable silent backbone of the specialty" and "a spare pair of hands." In 1996, it was shown that NCCGs in general were educationally undernourished, poorly represented, and precariously contracted. In 2005, has anything changed?

The GUM NCCG Group was formed in 2001 . The aim of the group is to work in partnership with consultants and all relevant professional bodies in order to represent the professional interests of all NCCG doctors, and to assist with the provision of grade relevant training. The group is affiliated to the British Association for Sexual Health and HIV (BASHH) and enjoys privileges similar to that of the special interest groups.

Various surveys have been undertaken via the NCCG Group, which include:

- 2001: Access to information technology and knowledge of guidelines; raising significant concern about the training and supervision of NCCGs in genitourinary medicine

- 2002: Study leave entitlement; showing that many NCCG doctors who are seeing a significant proportion of the clinical workload in GUM are not supported by their employing trusts to keep up to date.

- 2003: Job plans and appraisal; NCCGs are disadvantaged in terms of appraisal, continuing professional development (CPD) and career progression and some jobs will be at risk as revalidation approaches.

- 2004: Repeat survey of appraisal and study leave; showing that $34 \%$ of those who attended the national conference at York had never had their GUM work appraised either separately or as part of appraisal in another area. In addition, $21 \%$ had no GUM study leave entitlement.

These findings continue to give rise for concern, as all doctors require updating and development in order to provide quality care.

The Department of Health launched a consultation document in July 2003 entitled Choice and Opportunity-Modernising Careers for Non Consultant Career Grade Doctors.

The key principles and recommendations included:

- Clear criteria should govern entry to career grades

- Career pathways should be explicit

- Existing NCCG grades should be integrated into a single, simplified structure with no more than two recognised levels of practice

- Individual competencies of an NCCG should be assessed and formally recognised enabling them to work as independent practitioners within their area of competence

- CPD should be available according to the needs of NCCGs

- Formal opportunities for NCCGs to resume training should be available.

The response to the consultation exercise in England was reported in January 2004. The consultation was widely welcomed and many respondents commented on how well the recommendations captured key issues.

It will take time to implement Choice and Opportunity so what should the specialty be doing now? We believe that:

(1) There is a need to maintain an up to date accurate database of GUM NCCG doctors.

(2) The GUM aspect of NCCG doctors should be appraised regularly to ensure that GUM educational needs are met (although at present there is uncertainty as to what constitutes appropriate "specialty specific appraisal" for doctors who participate in appraisal mechanisms elsewhere).

(3) There should be NCCG representation on regional BASHH branch committees.

The BASHH Clinical Governance Committee is currently working together with the NCCG Group towards achieving these objectives. While it is an NHS trust responsibility to ensure that appraisal occurs, consultants and line managers need to be proactive now with regard to the appraisal function and ensure equity of CPD funding for NCCGs. Without CPD and appraisal, revalidation may not be possible and some NCCG doctors (who are essential providers of GUM services) might be lost.

R Challenor
GUM Department, Derriford Hospital, Plymouth
PL6 8DH, UK; rachel@challenor.biz
M R Pakianathan
Department of GUM, St George's Hospital,
London SWI7 OQI, UK

